\title{
Combinatorial Clock Auctions: Price Direction and Performance
}

\author{
David R. Munro* Stephen J. Rassenti ${ }^{\dagger}$
}

May 29, 2013

\begin{abstract}
This paper addresses three concerns with ascending price Combinatorial Clock Auctions (APCA); price guidance toward efficiency relevant packages, computational burden, and susceptibility to collusive bidding. We propose a descending price Combinatorial Clock Auction (DPCA) with a newly devised pricing strategy to alleviate all of these concerns. Mimicking bidding behavior of human subjects found in previous laboratory experiments, agent-based simulations of a DPCA show improvements in efficiency resulting from better price guidance and a reduction in computational burden when compared to an APCA.
\end{abstract}

Keywords: Combinatorial Auctions, Efficiency, Computational Burden.

JEL Codes: D44, D61

*Department of Economics, University of California Santa Cruz, 1156 High Street, Santa Cruz, CA, USA 95064. Corresponding author. Email: munro.david.r@gmail.com; Tel.: +1 (831) 4595617

${ }^{\dagger}$ Economic Science Institute, Chapman University, One University Drive, Orange, CA, USA 


\section{Introduction}

An important feature of any auction is its ability to generate an efficient allocation of goods. There exist many environments in which the goods being sold have complementary values when purchased in combinations. In these circumstances traditional single unit auctions can lead to inefficient allocations because of the risks associated with the exposure problem: when a bidder submits bids on several single-items in anticipation of achieving a complementary package value but then fails to win one or more of the requisite items. To combat this problem Rassenti et al. (1982) proposed a sealed bid combinatorial auction for the selling of airport landing and take-off slots in packages that were suitable for an airline interested in the efficient daily routings of its planes from airport to airport at particular times. This sealed bid combinatorial institution, however, places considerable burden on bidders. For example, in a combinatorial auction with only ten items, where a bidder has positive value for all individual items as well as any combination of them, there are $2^{10}-1$, or 1023 possible combinations for the bidder to place a bid on. In addition to the task of identifying and submitting all possible bids, is the daunting task of value elicitation. Determining ones value for an item and its possible synergistic value in combination with all possible other packages of items can be a very expensive and time-consuming process. This fact is not of trivial concern when designing auctions that will function in the real world.

This burden on bidders can be greatly reduced in an auction which provides price feedback. In recent years, several iterative combinatorial auction institutions have been designed to improve bidding saliency, auction efficiency, and seller revenue; Milgrom (2000) proposed The Simultaneous Ascending Auction (SAA) where goods are sold simultaneously with linear pricing and no package bids are accepted by the auctioneer, Porter et al. (2003) proposed the Combinatorial Clock Auction (CCA) also uses simultaneous linear prices but allows for package bids, Ausubel et al. (2004) proposes the two-stage clock-proxy auction which uses the SAA in the clock phase, and then abandons linear pricing in the proxy-bidding stage to enhance auction efficiency and revenue, and Goeree and Holt (2010) proposes the Hierarchical Package Bidding auction, where the auctioneer pre-selects packages available for 
bidding. Each of these institutions has strengths and weaknesses, and each are designed to help overcome specific issues. Evidence from laboratory and real world implementation has shown that these iterative auctions are able to achieve high levels of efficiency and revenue (see McAfee and McMillan (1996), Cramton (1997), Porter et al. (2003), Kwasnica et al. (2005), Brunner et al. (2010), and Kagel et al. (2010) among others).

Kagel et al. (2010) (henceforth KLM) test a variant of the CCA proposed in Porter et al. (2003) in a laboratory setting, and find the institution performs relatively well, albeit not as well as Porter et al. (2003), but uncover an important distinction in the auction environment that leads to a disparity in the institutional performance given the bidding behavior they observe. The bidding behavior observed is a myopic strategy where bidders consistently bid for their first and sometimes second most profitable package in each round of the auction. Given this bidding behavior KLM discover easy and hard auction environments. Easy environments are ones where myopic bidding leads to allocations that are $100 \%$ efficient. " "Hard" environments are ones where myopic bidding leads to allocations that are less than 100\% efficient. To achieve $100 \%$ efficiency in "hard" auctions bidders must bid on less profitable packages somewhere during the course auction, something myopic bidding fails to do. We will refer to this as the relevant package problem: when prices fail to direct bidders to their efficiency relevant packages. Given the bidding interface used in KLM, the myopic bidding found in these experiments is likely driven my strategic motives.

A different sort of myopic bidding is found in Scheffel et al. (2012). In laboratory testing of a number of iterative combinatorial auctions they find that the biggest hindrance of efficient allocations is not auction rules but the bidders' restricted package selection. Bidders pre-select packages in the first round of the auction and focus their bidding on this small subset of profitable packages throughout the auction. This is different from the myopic bidding in KLM because it involves limited switching of the packages that receive bidding attention. An important difference of this study from that of KLM is that bidders are not presented with a full summary of their potential package bids and must "elicit" package

\footnotetext{
${ }^{1}$ This measure of efficiency will be defined later in the paper.
} 
values through a search process. Because Scheffel et al. (2012) observe that bidders elicit a very small percentage of their potential packages they posit that the static myopic bidding they observe is a result of bidders cognitive limits rather than strategic motives.

Aside from this observed restrictive bidding and the associated relevant package problem there are two other main concerns with iterative combinatorial auctions. The first is the potential computational burden associated with running them. In order for an auction to function appropriately it must have the ability to be completed in a reasonable amount of time. Due to the potential for complex overlapping of package and single-item bids in a combinatorial auction environment the dimensions of the branching tree associated with the optimizations embedded in the these auctions can be substantial, creating uncertainty in computation time. One solution to excessive computation times is to reduce the number of optimization branches requiring examination through a restriction of the bidding space which can eliminate complex overlapping bids. This can be achieved through shifting the computational burden onto bidders, forcing them to solve which bids will combine optimally with other stand-by bids to displace current winning bids as in Banks et al. (1989), or by prepackaging the items that bidders are allowed to submit bids on as in Goeree and Holt (2010). Both methods may be undesirable. The first generates substantial bidding complexity and the second relies on the auctioneer to predict decentralized information regarding desirable bundles of items. ${ }^{2}$

The second major concern with iterative combinatorial auctions is the susceptibility of ascending multi-unit auction institutions to collusive bidding. While collusion has not been a main focus in combinatorial auction research there is a rich literature on collusion in non-package multi-unit ascending price institutions.

In this paper we propose that a novel Descending Price Combinatorial Auction, henceforth DPCA, which will ameliorate all of the problems mentioned above. Namely, it will better guide bidders to reveal their efficiency relevant packages, limit computation time by reducing the number of bids submitted to successive the auction optimizations, and prevent

\footnotetext{
${ }^{2}$ For more discussion on computational issues and combinatorial auctions see Rothkopf et al. (1998) and Fujishima et al. (1999)
} 
bid signaling and retaliatory bidding that lead to collusive behavior. From the running of agent-based simulations in an environment that matches that of KLM, we find substantial improvement on the relevant package problem and the computational burden problem. From preceding literature we summarize evidence of collusion in multi-unit ascending auctions and present the evidence for the collusion breaking power of descending price multi-unit auctions.

The remainder of this paper will be structured as follows; Section 2 will summarize the relevant work completed on ascending price Combinatorial Clock Auctions, Section 3 will formalize the rules of the DPCA, Section 4 will summarize the testing undertaken using agent-based simulations, present the results from the simulations, and summarize the support for descending multi-unit institutions as a means to prevent collusion, and finally Section 5 will summarize our conclusions.

\section{Ascending Price Combinatorial Auction Overview}

To distinguish between the price direction of the two auctions we discuss in this paper we will refer to the CCA proposed by Porter et al. (2003) as the ascending price Combinatorial Clock Auction (APCA). The APCA version tested in KLM works in the following manner. Prices for each item start at levels set by the auctioneer. In any given round bidders are free to bid on any and all combinations of items or on single-items. If an item receives two or more new bids, or if an item named in a provisionally winning bid is included in a different new bid, then the items price is raised. At the end of each round the auctioneer runs an optimization to determine which bids generate the highest revenue and it is these bids that become the provisionally winning bids. When there is a round where no new bids are placed, and hence no price increases, the auction is over. ${ }^{3}$

The differences between this auction and the Porter et al. (2003) APCA are the use of the XOR bidding rule, the announcement of provisional winners in each round of the auction,

\footnotetext{
${ }^{3}$ KLM uses a rule of two rounds with no bidding activity to determine the end of the auction. We leave this rule out because we are using agent-based simulations.
} 
the auction ending rule, and the pricing mechanism. ${ }^{4}$ The XOR rule is a constraint that allows the auctioneer to select at most one bid from each bidder when allocating goods. This rule is presumably used to reduce some of the bidding complexity for bidders because they do not have to worry about having multiple bids accepted, but it also adds a significant strategic component to bidding.

\subsection{APCA Environment}

To allow us to piggyback on the laboratory behavior discovered in KLM, we employ the same auction environment. A graphical representation can be seen in Figure 1.

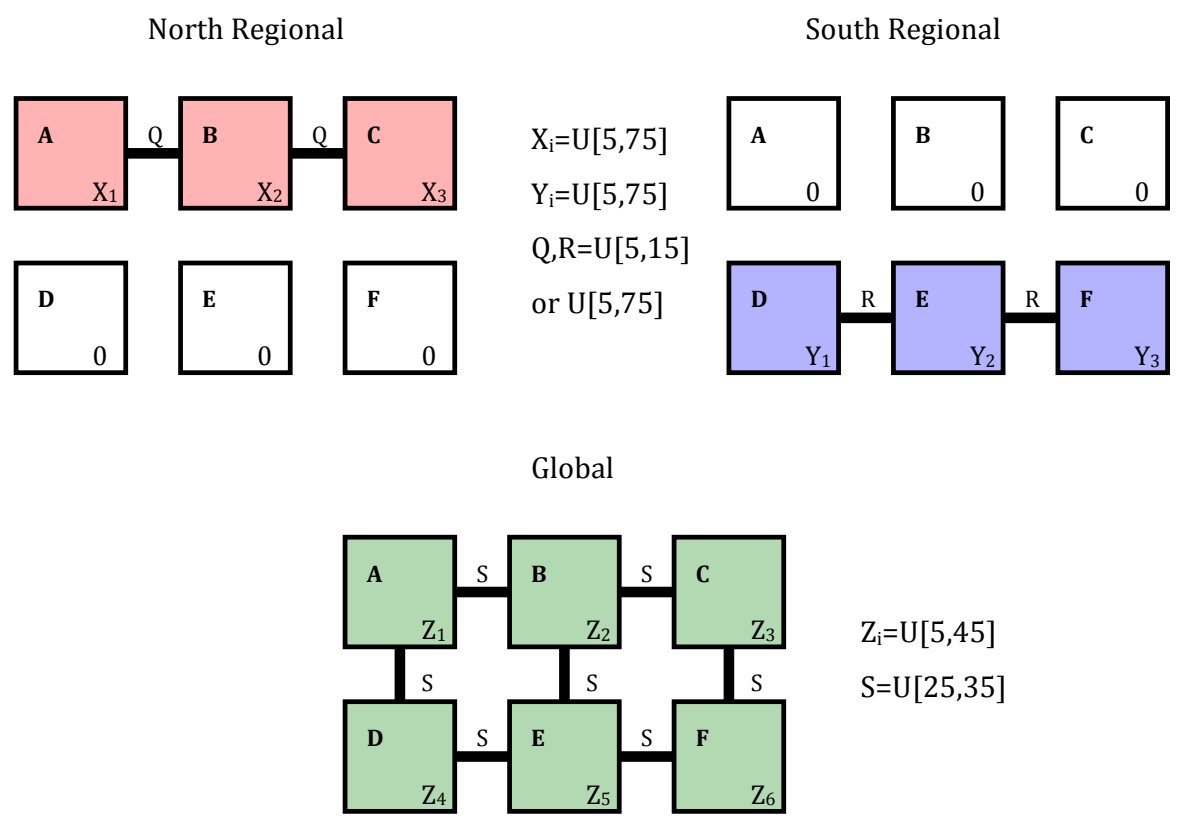

Figure 1: Diagram of item values and synergies for 6-item environment.

There are three bidders in this environment and either 4 or 6 items. Figure 1 displays the 6-item environment. The 4-item environment is the same but with items $\mathrm{C}$ and $\mathrm{F}$ removed. In these environments the Regional bidders have items values randomly drawn from the

\footnotetext{
${ }^{4}$ The announcement of provisionally winning bids in each round does not affect our simulations because we did not incorporate it into agent behavior. To match the KLM environment we incorporate their ending rule, pricing mechanism, and XOR constraint into our simulations.
} 
uniform distribution [5, 75], and Global bidders have item values randomly drawn from the uniform distribution $[5,45]$. Package values are created by the summation of the item values included in that package and the synergies that exist between adjacent items. For example, in the six item auction the North Regional bidders package AB would be equal to: $\mathrm{X} 1+\mathrm{X} 2$ $+\mathrm{Q}$, where $\mathrm{X} 1$ is the value for item $\mathrm{A}, \mathrm{X} 2$ is the value for item $\mathrm{B}$, and $\mathrm{Q}$ is the synergy value obtained when both items are won together. In this environment, the package AC would be equal to: $\mathrm{X} 1+\mathrm{X} 3$, because only adjacent items produce synergies.

There are two synergy environments. In the low synergy environment, Regional bidders draw their synergy values from the uniform distribution [5, 15] and in the high synergy environment they draw from the uniform distribution [25, 35]. The Global bidder always draws their synergy value from the uniform distribution [25, 35].

\subsection{Efficiency Relevant Packages}

If an auction has a unique efficient goods assignment, there exists a unique set of efficiency relevant packages/items. Formally, KLM define it as follows:

Definition 1 In a total-bid maximizing package auction, let $\beta$ denote the final bids in the auction and $\left(x^{a}, \tau\right)$ the auction outcome. If for all bidders $i, v_{i}\left(x_{i}(N)\right)-\beta_{i}\left(x_{i}(N)\right) \leq v_{i}\left(x_{i}^{a}\right)-$

$\beta_{i}\left(x_{i}^{a}\right)$, then the goods assignment $x^{a}$ is efficient: $\sum_{i=1}^{N} v_{i}\left(x_{i}^{a}\right)=w(N)$. If the goods assignment is unique, then the condition $v_{i}\left(x_{i}(N)\right)-\beta_{i}\left(x_{i}(N)\right) \leq v_{i}\left(x_{i}^{a}\right)-\beta_{i}\left(x_{i}^{a}\right)$ is necessary as well as sufficient for $x^{a}$ to be efficient.

As prices need not provide an indication of efficiency relevant packages, the sufficient condition required to fulfill $v_{i}\left(x_{i}(N)\right)-\beta_{i}\left(x_{i}(N)\right) \leq v_{i}\left(x_{i}^{a}\right)-\beta_{i}\left(x_{i}^{a}\right)$ in an ascending institution is for participants to submit bids for all packages where their price of package $j$ is less than bidder i's value for that package in each round of the auction. ${ }^{5}$ This is the source of the sub-efficient allocations in "hard" environments in KLM; bidders failed to submit bids on

\footnotetext{
${ }^{5}$ Only if price increments were small enough to divide the bids of close valuations into separate rounds would this behavior guarantee an efficient allocation.
} 
profitable, efficiency relevant, packages early in the auction and by the time the price vector indicated to bidders which packages were their relevant ones prices were too high to submit a bid on them. When bidders are not fully revealing, the APCA can therefore lead to inefficient allocations.

Using simulations with myopic bidding agents KLM discover which valuation/synergy environments are easy, producing an outcome that is $100 \%$ efficient, and environments that are hard, producing an outcome that leads to lower than $100 \%$ efficiency. They use these simulation results to choose which environments to test in the laboratory with incentivized subjects.

\subsection{Previous Results}

Testing these environments in the laboratory KLM find that bidders do follow a myopic bidding strategy and submit bids on many fewer packages than the total number of packages available to them. A bidders most profitable package attracts the most attention, while the second most profitable attracts considerably less attention. In addition, they find that the same behavior is displayed across the easy and hard environments. ${ }^{6}$

Given the relative ease of bidding in these laboratory auctions (bidders know precisely all of their values and can trivially submit multiple package bids ${ }^{7}$ ) it seems likely that bidders are employing some strategy in this myopic bidding. These strategies may coincide with the bidder's knowledge of the provisional winners and may be an attempt to prevent the auctioneer from using the XOR constraint to allocate anything but their best package at a particular vector of prices. It is important to note though is that whether these laboratory subjects are strategically under-revealing or not, bidders in the real world are likely to employ some degree of myopic behavior when faced with the task of value elicitation. Indeed, in a laboratory test of an ACPA where subjects must uncover their values through a search

\footnotetext{
${ }^{6}$ They cannot reject the null hypotheses that bidding is directed to the most profitable and second most profitable packages with equal frequencies in the easy and hard environments.

${ }^{7}$ All information regarding packages and items are presented to bidders in a very salient table and in order to submit a bid one simply needs to check a box.
} 
process Scheffel et al. (2012) finds that subjects choose a small subset of packages to bid on early in the auction and that this is the main hindrance in achieve efficient allocations. The purpose of using clock auctions in these multi-unit auctions is to reduce the burden on bidders, which explicitly means that one expects them to submit fewer bids to the auction than the total number of profitable bids available to them. Thus the failure of prices to guide bidders to efficiency relevant packages observed in KLM and Scheffel et al. (2012) are important considerations when attempting to design a combinatorial clock institution that leads to efficient allocations.

In this paper we appeal to the similarities observed in KLM between the bidding behavior in their laboratory experiments and that encoded in their simulations. They find that the performance of simulations with myopic bidders can qualitatively predict the distinction of easy and hard environments with human subjects.

\section{Descending Price Combinatorial Auction}

As a descending price auction the DPCA item prices begin at levels set by the auctioneer and decrement until the market cleared. However, the combinatorial environment presents difficulties regarding how to implement a value-maximizing price decrementing and stopping rule. In the DPCA if an item price strictly stopped decrementing as soon any bid was submitted that included that item, significant blocking of potentially efficient allocations would occur. On the other hand, stopping prices for highly valued items at high levels produces the price signals needed to guide participants to their efficiency relevant packages. DPCA employs a mechanism that intelligently brakes prices decrementing. Figure 2 displays a flow chart of the DCPA mechanism.

After each round a primary optimization is run to determine the optimal allocation of items given all of the bids previously submitted and the XOR constraint applicable to each bidder. If the market clears from this allocation (all items sell) then the auction is over. (Notice, a universal bid on all items ends the auction!) If the market does not clear then 


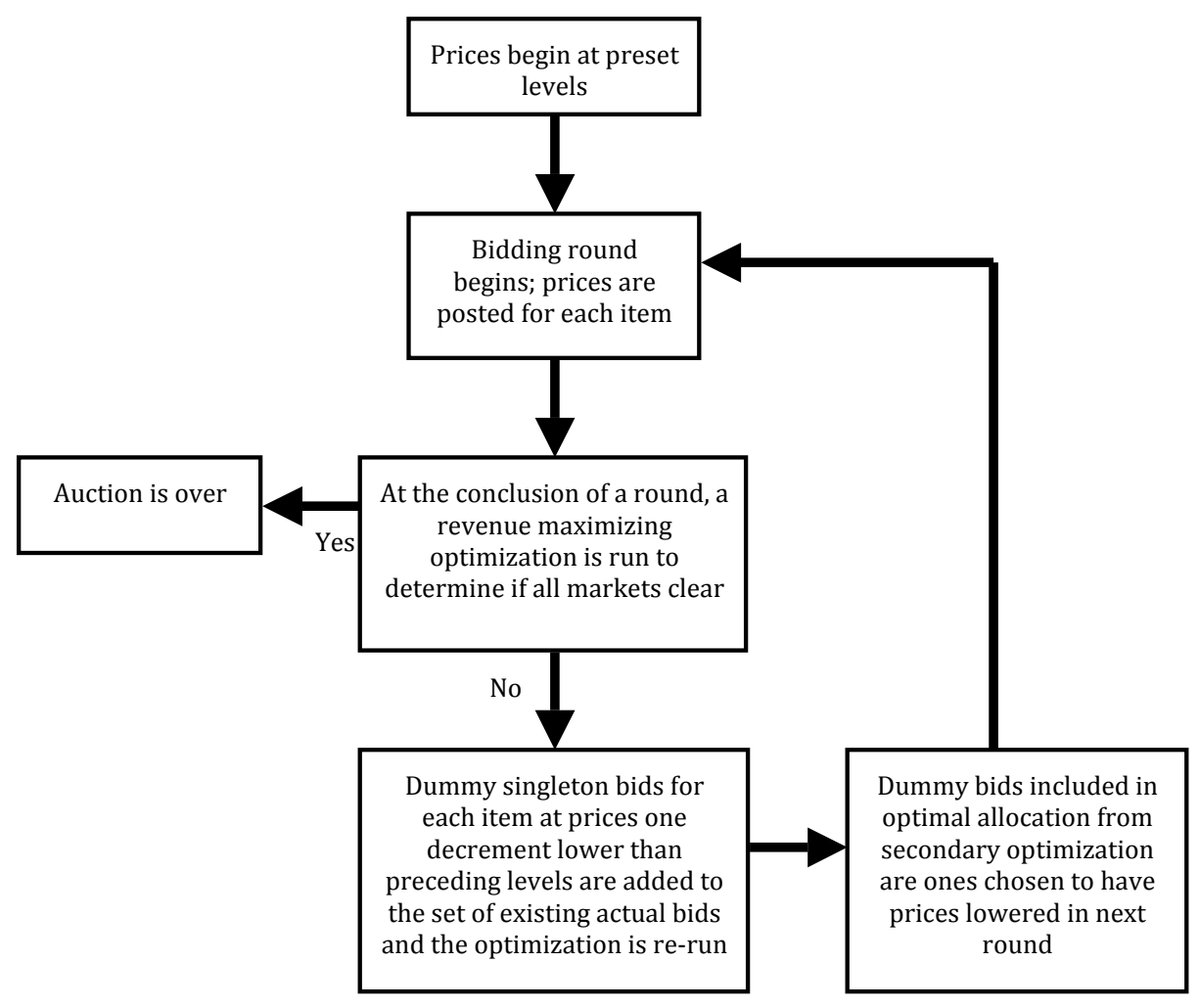

Figure 2: Flow chart of DPCA mechanism

some price(s) must be decremented. The price decrementing mechanism works as follows; in every round where the market does not clear and at least one bid has been initially submitted, a secondary optimization is run to determine the best prices to decrease. ${ }^{8}$ The secondary optimization simply embellishes the primary optimization by adding to it a set of "dummy bids for all single-items that could possibly be submitted in the next round at the next decremented price levels. Although the primary optimization did not clear the market, the optimal allocation resulting from this secondary pricing optimization (shown below) will always clear the market and include one or more of the dummy single-item bids at the tentatively decremented prices. Whichever single-item bids are included in this allocation are the items that are selected to have their prices decremented in the next round.

\footnotetext{
${ }^{8}$ There is no need to run the optimization until the first bid is submitted to the auction. Until that point all prices fall in unison, though perhaps at different rates.
} 


$$
\begin{array}{ll}
\underset{x_{i j}}{\operatorname{maximize}} & \sum_{i=1}^{N} \sum_{j=1}^{J} p_{j} x_{i j} \\
\text { subject to } & \sum_{i=1}^{N} \sum_{j=1}^{J} a_{i j k} x_{i j} \leq 1, \\
& \sum_{j=1}^{J} x_{i j} \leq 1, \\
& x_{i j}, a_{i j k} \in\{0,1\} .
\end{array}
$$

Where $p_{i j}$ is the price associated with each bid $x$, for bidder $i^{\prime} s$ package $j$ and where $a_{i j k}$ indicates which items $k$ are included in bidder $i^{\prime} s$ package $j$. Given this pricing rule prices on all items included in any non-dummy bids accepted as winners by the secondary optimization will temporarily stop decrementing. This intelligent price-braking, which encourages the submission of the missing bids with the greatest potential to be efficiency relevant, is key to the DPCAs effectiveness.

\subsection{Auction Environment}

To allow us to draw useful comparisons to the results reported in both the laboratory and simulation testing completed by KLM we construct the same simulation environment outlined in Section 2. To test efficiencies in both institutions we employ both a 4-item and 6-item environment with three bidders in each and we randomly draw values and synergies from the uniform distributions specified above. In addition to the environment studied in KLM, we also study the same environments, but with double the bidders, i.e. two north regional bidders, two south regional bidders, and two global bidders.

For the simulations we construct twenty package files that include all the possible packages for each regional and global bidder and randomly draw the synergies corresponding to those packages. We also construct fifty value files that draw random item values for all three bidders. Thus when we combine all package and value files we have a thousand distinct auction environments. These environments are static across the paired four or six item, high 
or low synergy, ascending or descending auctions.

\subsection{Bidding Strategy}

The obvious question when thinking about a descending version of a combinatorial clock auction is what strategy should the bidders be implementing? Vickrey (1961) solves the theory for the optimal bid, $B_{i}$, in a single-item sealed bid (or descending) auction:

$$
B_{i}=\frac{N-1}{N} V_{i}
$$

Where, $N$ is the number of bidders in the auction and $V_{i}$ is the private value for bidder $i$. The bidding strategy in combinatorial environments becomes much more complicated. While it has not been theoretically proven, the Bayesian Nash Equilibrium bid shave in a DPCA likely depends on the number of bidders in the market competing for each item a bidder is interested in, what packages those competing bidders are interested in, the number of bidders in the market for the items in those packages, the values/synergies of the packages you are interest in, the values/synergies of your competitors for those items, and the values/synergies of their competitors on other items.

This paper is not focused on optimal bidding strategies, but on a comparison between auction formats. However, in order to accurately comment on the performance of auctions, specifically on the efficiency of allocation, we need to make them close to theoretically equivalent. We rely on results from the APCA simulations to do this. In the easy APCA simulation results, we can compute the difference between the selling prices of each package/item and each winning bidders private value. Finding the average bid shave (winning price/valuation) for each winning bidder over numerous auctions will provide a close estimate to the theoretically optimal bid shave that would make these auctions revenue equivalent. ${ }^{9}$ Because the optimal bid shave may be different for regional as opposed to global bidders, we compute the

\footnotetext{
${ }^{9}$ Consider the following intuitive example of a second price single unit auction where values are drawn randomly from a uniform distribution. If the bidders are bidding their value in a second price auction, then the computed average bid shave for the first price auction will converge toward $\frac{N-1}{N} V_{i}$.
} 
average bid shave separately for each bidder and use these as the bid shaves for the bidders in the matched DPCA environment.

\subsection{Efficiency}

We compute efficiency in the following manner,

$$
\text { Efficiency }=\frac{V_{a}-V_{\text {random }}}{V^{*}-V_{\text {random }}}
$$

Where, $V_{a}$ is the sum of the private values for the winners of the auction, $V^{*}$ is the sum of the values of the efficiency maximizing allocation in the system, and $V_{\text {random }}$ is derived by the mean values from 1000 random feasible allocations of items to bidders with positive values in each of the 1000 value/package environments. This procedure was followed for each distinct environment.

\subsection{Simulations}

The simulations we employ are straightforward. For each of the environments constructed the agents are assigned their item values, packages, and synergies to compute their package values. In each round of the auction the myopic agents take the vector of prices and compute their most profitable package and submit a bid only for that package. All other packages receive no bids. We record all of the bids throughout the simulations and compute the efficiency of each environment for both the APCA and DPCA using the winning bids of the auctions.

\subsection{Hypotheses}

Using a descending vector of prices we expect the DPCA to produce higher efficiencies than the APCA given myopic bidding. As prices fall in the DPCA agents will submit bids on their most efficiency relevant packages first, stopping or "slowing those prices, making those items out of reach for non-efficiency relevant packages. The items with prices that continue falling 
will become the more profitable and attract efficiency relevant bids to items where resolving the combinatorial misfit problems promise the strongest gain. The DPCA will direct bidders toward those efficiency relevant packages/items while avoiding the price blocking problem encountered in the APCA because bidders can always make bids on packages/items that may not be their highest valued. Of course, the DPCA prices will not be a perfect guide to bidders because we set all prices to start at some arbitrary level and decrement at a pre-fixed rate. Some improvement could no doubt be made in dynamically tempering the pricing algorithm, just as could be in the APCA, but we chose to compare them in their simplest common implementation. Thus we do not expect the DPCA to produce an absolute case-by-case improvement in efficiency, but we do expect a meaningful improvement in the average efficiency produced.

The computation time associated with combinatorial auctions is a concern when attempting to implement an auction on a reasonable amount of time. Integer optimizations are part of both the ascending and descending institutions. The larger the objective function (more items and packages) in these optimizations, the longer they will take to solve. In ascending auctions the auctioneer sets the starting price at some level below where she believes the final item sale prices will be. As a consequence the CCA picks up many bids along the way that are irrelevant to the efficient allocation because packages/items would only be profitable for many bidders at low prices. In a descending environment, however, prices are set above where the auctioneer believes the final item sale prices will be. As the prices reach levels where it becomes profitable for participants to submit a bid it is much more likely that many of those bids are going to be efficiency relevant. We time each of the thousand auctions under each institution. The APCA contains only one optimization for each round of the auction, the one that determines if the market is cleared and who the provisional winners are. The DPCA contains two optimizations per round, the first to test for market clearing and the second (a slight embellishment for which the first provides an excellent starting solution) to determine new prices, but these optimizations need only be run once the original bid has 
been submitted to the system. ${ }^{10}$ Under myopic bidding behavior it is expected that there will be little difference in computation time in the ascending and descending institutions, simply because there is only one bid from each bidder in each round and the calculations are computationally trivial for modern codes and hardware. Therefore we also tested an environment where bidders employ an all-revealing strategy; a bid is submitted on all profitable packages in every round of the auction. We hypotheses that the auction run times will be slower for the APCA than the DPCA when agents are bidding in an all revealing fashion because of the relative increase in size of the objective function caused by ascending prices. ${ }^{11}$ To test how computational time grows with increased numbers of bidders and items we construct larger auction environments. In addition to the 4-item and 6-item environment KLM use we construct a 12-item environment. Also, as above, we double the competition from three to six bidders. In the environment where agents are fully revealing we expect the disparities between the APCA and DPCA to grow with both the number of bidders, and the number of items in the auction.

\section{Testing}

As mentioned above a thousand unique value/package environments are tested in the APCA and repeated in the DPCA. To test the extent of the relevant package problem we run those environments with myopic bidding behavior in the matching versions of the ascending/descending institutions. Again, there are 4 unique value/package environments for both the three and six-bidder environments. One for the low synergy 4-item, one for the high synergy 4-item auction, one for the low synergy 6-item auction, and finally one for the high

\footnotetext{
${ }^{10}$ We time the entire length of each auction for this reason. This however means that background code running setup and database retrieval and storage in the simulations is also timed. This piece of the code, however, is the identical for both the APCA and the DPCA. Thus, the important fact in the computation times reported below is not so much the absolute value of the computation time, but the relative differences between the ascending and descending institution.

${ }^{11}$ Some preprocessing of bids prior to optimization could reduce computation time, however institutional rules can prevent significant shortening of objective functions. For example, the XOR constraint would prevent one from eliminating dominated bids from the objective function because the system is restricted to taking only one bid from each participant.
} 
synergy 6-item auction.

Besides the complex problem of choosing what packages/items to bid on and when in a APCA, the bidding task is quite simple: continue to bid on a package or item until the price passes your value. This feature of ascending auctions makes them less susceptible to "noisy bidding behavior, where a bidder strays from the theoretically optimal second price auction bidding strategy. However, because theoretically optimal bidding in a descending auction requires deciding how far to let prices drop before submitting a bid, there can be significant noise detected in bidding.

In order to test the robustness of the efficiencies found in the DPCA, we incorporate some noise into the simulated agents bidding function. To use a reasonable measure of noisy bidding we take the laboratory data from first price auctions in Harrison (1989). Those familiar with the experimental first price auction literature are aware that most laboratory subjects have a strong tendency to overbid. This overbidding is typically explained by some measure of risk aversion. We assume no overbidding in our DPCA simulations (risk aversion would improve its revenue performance) and are more interested in getting a reasonable measure of the noise present in recorded bidding behavior. We use the standard deviations of first price bids reported by Harrison (1989) to test the robustness of the DPCA. To program this noisy bidding behavior into our agents we construct a beta distribution for each bidder with a mean bid shave corresponding to the amounts found from the easy ascending auctions and a standard deviation from the Harrison (1989) results. ${ }^{12}$ Therefore, in every auction every bidding agent draws once from his beta distribution and applies this bid shaving strategy to all bids on all items/packages during that auction.

\subsection{Results: Relevant Package Problem}

As hypothesized, the DPCA does not suffer as much as the APCA from lower efficiencies under myopic bidding because descending prices better guide bidders to their efficiency

\footnotetext{
${ }^{12}$ From the Harrison (1989) results we use the average standard deviation from the assumed risk neutral cases and we do not use data from the experiments where subjects are bidding against a RNNE bidder.
} 
relevant packages. In all environments without noisy bidding, and all but one environment with noisy bidding, the DPCA improves on the APCA in terms of higher average efficiencies and a higher number of allocations that are 100\% efficient. Table 1 reports the results from the three-bidder ascending and descending clock auctions, Table 2 reports the statistical testing from the three-bidder environment, Table 3 reports the efficiency results from the six-bidder ascending and descending clock auctions, and finally Table 4 reports the statistical testing from the six-bidder environment.

\begin{tabular}{|c|c|c|c|c|c|}
\hline & & & $\begin{array}{c}\text { APCA } \\
\text { Efficiency }\end{array}$ & $\begin{array}{c}\text { DPCA } \\
\text { Efficiency } \\
\text { (no error) } \\
\end{array}$ & $\begin{array}{c}\text { DPCA } \\
\text { Efficiency } \\
\text { (Beta error*) }\end{array}$ \\
\hline \multirow[t]{4}{*}{ 4-Item Auction } & \multirow[t]{2}{*}{ Low Synergy } & $\begin{array}{c}\text { Mean } \\
\text { (std. error) }\end{array}$ & $\begin{array}{c}90.69 \% \\
(22.91 \%)\end{array}$ & $\begin{array}{c}96.73 \% \\
(10.96 \%)\end{array}$ & $\begin{array}{c}95.06 \% \\
(15.38 \%)\end{array}$ \\
\hline & & $\begin{array}{l}\% \text { of auctions } \\
100 \% \text { efficient }\end{array}$ & $74.10 \%$ & $84.20 \%$ & $80.90 \%$ \\
\hline & \multirow[t]{2}{*}{ High Synergy } & $\begin{array}{c}\text { Mean } \\
\text { (std. error) }\end{array}$ & $\begin{array}{c}91.15 \% \\
(20.40 \%)\end{array}$ & $\begin{array}{l}98.90 \% \\
(5.93 \%)\end{array}$ & $\begin{array}{c}96.27 \% \\
(14.87 \%)\end{array}$ \\
\hline & & $\begin{array}{l}\% \text { of auctions } \\
100 \% \text { efficient }\end{array}$ & $75.90 \%$ & $91.60 \%$ & $85.30 \%$ \\
\hline \multirow[t]{4}{*}{ 6-Item Auction } & \multirow[t]{2}{*}{ Low Synergy } & $\begin{array}{c}\text { Mean } \\
\text { (std. error) }\end{array}$ & $\begin{array}{c}95.65 \% \\
(13.33 \%)\end{array}$ & $\begin{array}{c}97.36 \% \\
(10.04 \%)\end{array}$ & $\begin{array}{c}96.51 \% \\
(12.16 \%)\end{array}$ \\
\hline & & $\begin{array}{l}\% \text { of auctions } \\
100 \% \text { efficient }\end{array}$ & $78.80 \%$ & $82.30 \%$ & $81.20 \%$ \\
\hline & \multirow[t]{2}{*}{ High Synergy } & $\begin{array}{c}\text { Mean } \\
\text { (std. error) }\end{array}$ & $\begin{array}{c}90.89 \% \\
(17.41 \%)\end{array}$ & $\begin{array}{l}98.03 \% \\
(5.97 \%)\end{array}$ & $\begin{array}{c}95.45 \% \\
(11.64 \%)\end{array}$ \\
\hline & & $\begin{array}{l}\% \text { of auctions } \\
100 \% \text { efficient }\end{array}$ & $66.50 \%$ & $83.80 \%$ & $76.60 \%$ \\
\hline
\end{tabular}

Table 1: Three-Bidder Auction Efficiencies

When the agents in the DPCA are equipped with noisy bidding functions, the average 


\begin{tabular}{|c|c|c|c|}
\hline & \multicolumn{2}{|c|}{ Statistical Significance } & \multirow{2}{*}{$\begin{array}{c}\text { Practical Significance } \\
\text { Effect size }\end{array}$} \\
\hline $\begin{array}{l}\text { Paired } \\
\text { Environments }\end{array}$ & $\begin{array}{c}\text { Z-score } \\
\text { approximations }\end{array}$ & $\begin{array}{c}\text { p-value } \\
\text { (two-tailed) }\end{array}$ & \\
\hline 4-Item Low Synergy & -9.67 & 0.00 & 0.29 \\
\hline 4-Item High Synergy & -12.03 & 0.00 & 0.39 \\
\hline 6-Item Low Synergy & -5.81 & 0.00 & 0.17 \\
\hline 6-Item High Synergy & -12.90 & 0.00 & 0.42 \\
\hline 4-Item Low Synergy* & -6.21 & 0.00 & 0.19 \\
\hline 4-Item High Synergy* & -7.97 & 0.00 & 0.22 \\
\hline 6-Item Low Synergy* & -3.14 & 0.00 & 0.08 \\
\hline 6-Item High Synergy* & -7.97 & 0.00 & 0.26 \\
\hline \multicolumn{4}{|c|}{$\begin{array}{l}\text { * indicates environments where the descending auction in the matched pair incorporates } \\
\text { noisy bidding. z-score approximations reported from Wilcoxon Signed-Rank test, where } \\
H_{0}: \text { APCA Efficiency=DPCA Efficiency. } \\
\text { Effect size }=\left|\frac{\text { mean of paired difference in efficiencies }}{\text { standard deviation of paired difference in efficiencies }}\right|\end{array}$} \\
\hline
\end{tabular}

Table 2: Statistical Testing for Three-Bidder Results

efficiency of the allocations falls, but still significantly outperforms the APCA in both the average efficiency and number of auctions reaching 100\% efficiency in all but the six-bidder six-item low synergy environment where a myriad of nearly optimal solutions exists. These results confirm the hypothesis that the prices in the DPCA will better guide bidders to their efficiency relevant packages.

In addition, we also find that the difference in performance between the APCA and DPCA is greatest in the high synergy environments. This is likely due to the fact that in the low synergy environments the added value of efficiency relevant price guidance is much lower than in high synergy environments where there exists an increased probability that, if it can be found, a unique combination of high synergy bids from various bidders can produce a significant increase in surplus generated. Clearly major consideration should be given to implementing a DPCA when high synergies are a feature of the bidding environment. 


\begin{tabular}{|c|c|c|c|c|c|}
\hline & & & $\begin{array}{c}\text { APCA } \\
\text { Efficiency }\end{array}$ & $\begin{array}{c}\text { DPCA } \\
\text { Efficiency } \\
\text { (no error) }\end{array}$ & $\begin{array}{c}\text { DPCA } \\
\text { Efficiency } \\
\text { (Beta error*) }\end{array}$ \\
\hline \multirow[t]{4}{*}{ 4x2-Item Auction } & \multirow[t]{2}{*}{ Low Synergy } & $\begin{array}{c}\text { Mean } \\
\text { (std. error) }\end{array}$ & $\begin{array}{l}95.10 \% \\
(9.68 \%)\end{array}$ & $\begin{array}{l}98.48 \% \\
(3.69 \%)\end{array}$ & $\begin{array}{l}96.07 \% \\
(8.16 \%)\end{array}$ \\
\hline & & $\begin{array}{l}\% \text { of auctions } \\
100 \% \text { efficient }\end{array}$ & $67.10 \%$ & $79.10 \%$ & $69.30 \%$ \\
\hline & \multirow[t]{2}{*}{ High Synergy } & $\begin{array}{c}\text { Mean } \\
\text { (std. error) }\end{array}$ & $\begin{array}{l}96.42 \% \\
(8.08 \%)\end{array}$ & $\begin{array}{l}99.48 \% \\
(2.74 \%)\end{array}$ & $\begin{array}{l}97.18 \% \\
(7.06 \%)\end{array}$ \\
\hline & & $\begin{array}{l}\% \text { of auctions } \\
100 \% \text { efficient }\end{array}$ & $74.30 \%$ & $87.70 \%$ & $76.80 \%$ \\
\hline \multirow[t]{4}{*}{ 6-Item Auction } & \multirow[t]{2}{*}{ Low Synergy } & $\begin{array}{c}\text { Mean } \\
\text { (std. error) }\end{array}$ & $\begin{array}{l}97.48 \% \\
(6.09 \%)\end{array}$ & $\begin{array}{l}98.14 \% \\
(4.73 \%)\end{array}$ & $\begin{array}{l}96.59 \% \\
(7.63 \%)\end{array}$ \\
\hline & & $\begin{array}{l}\% \text { of auctions } \\
100 \% \text { efficient }\end{array}$ & $73.90 \%$ & $75.80 \%$ & $70.20 \%$ \\
\hline & \multirow[t]{2}{*}{ High Synergy } & $\begin{array}{c}\text { Mean } \\
\text { (std. error) }\end{array}$ & $\begin{array}{c}92.97 \% \\
(11.00 \%)\end{array}$ & $\begin{array}{l}98.43 \% \\
(3.74 \%)\end{array}$ & $\begin{array}{l}96.32 \% \\
(7.38 \%)\end{array}$ \\
\hline & & $\begin{array}{l}\% \text { of auctions } \\
100 \% \text { efficient }\end{array}$ & $54.80 \%$ & $75.10 \%$ & $64.00 \%$ \\
\hline
\end{tabular}

\footnotetext{
* indicates simulation where bids are drawn from a beta distribution with mean corresponding to bid-shave found in easy ascending auctions and standard deviation take from Harrison (1989), std. $\operatorname{dev}=0.0683$
}

Table 3: Six-Bidder Auction Efficiencies

\subsection{Results: Computational Burden}

Table 5 and Table 6 report the average auction time for each institution under both the myopic and reveal all strategies for the three-bidder and six-bidder environments respectively.

The myopic bidding environment shows little difference in average auction computation time between the APCA and the DPCA, column 1 and 2 in Table 5 and Table 6 provide the comparisons. ${ }^{13}$ When more bids are placed in the system under the reveal-all strategy the

\footnotetext{
${ }^{13}$ The increased time in both the APCA and DPCA myopic bidding environments in the larger auctions is a result of the increased time required to run the background code in the simulations. As mentioned earlier, this background code is the same for both institutions, which is why the relative difference in computation
} 


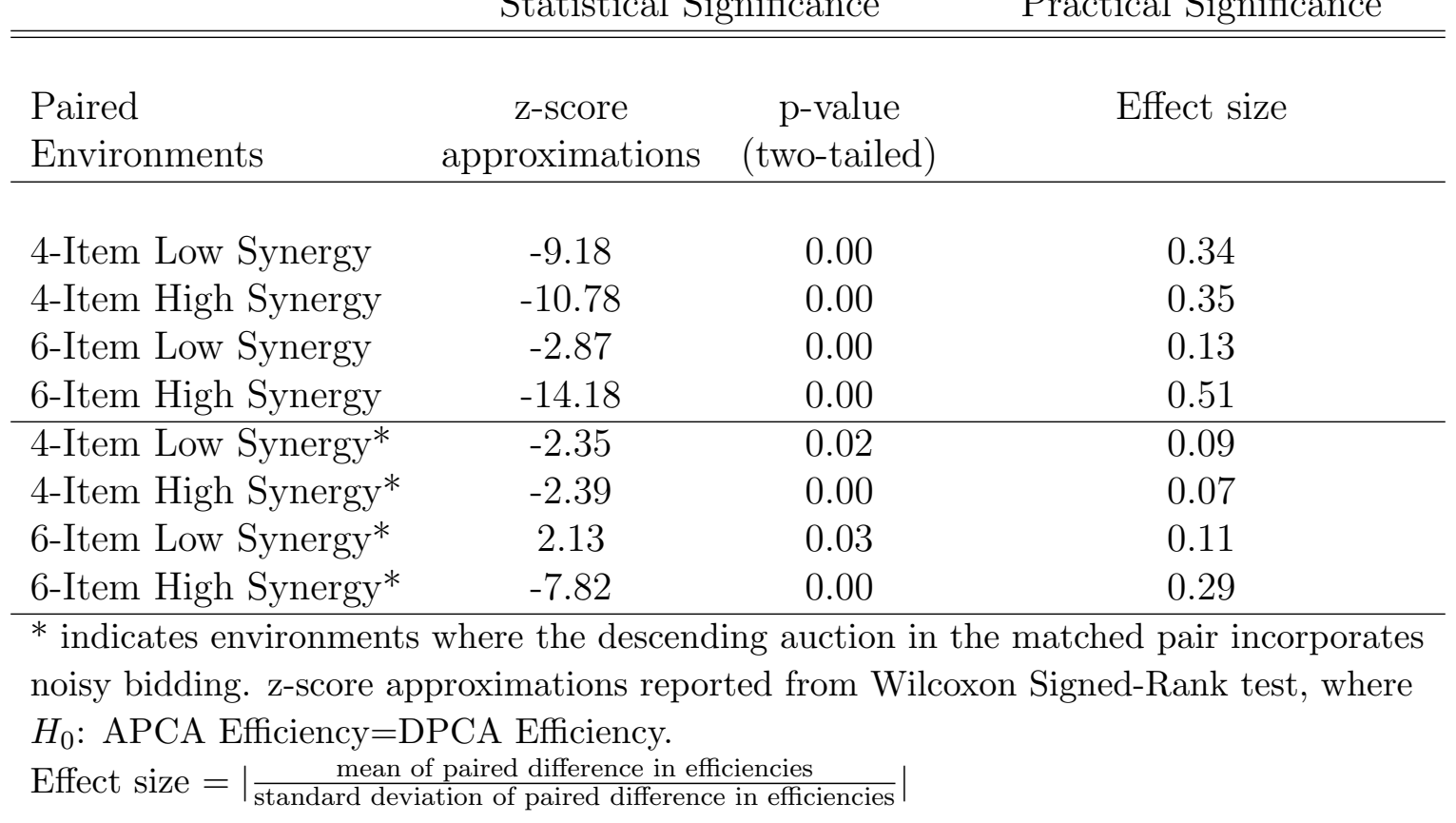

Table 4: Statistical Testing for Six-Bidder Results

APCA slows significantly in contrast to the DPCA, column 3 and 4 in Table 5 and Table 6 provide the comparisons.

In the three-bidder reveal-all environment, the APCA average auction computation time goes from a minimum of 0.156 seconds to a maximum of 90.38 seconds, an increase by a factor of 580. Where the DPCA average auction computation time goes from a minimum of 0.055 seconds to a maximum of 0.818 seconds, an increase by a factor of 15 . In the six-bidder reveal-all environment, the APCA average auction computation time goes from a minimum of 0.304 seconds to a maximum of 571.55 seconds, and increase by a factor of 1880 . Where the DPCA average auction computation time goes from a minimum of 0.067 seconds to a maximum of 1.526 seconds, an increase by a factor of 23 . Only some of the computation times in Tables 5 and 6 pose any real concern for an auctioneer, as the environments tested are quite small, but results are intended to be indicative of how the computation time required in the APCA grows, non-linearly, as the number of bidders and items grow.

times between the APCA and DPCA remain unchanged with myopic bidding in the larger environments 
Time take to complete auction (measured in seconds)

Myopic bidding Reveal-all bidding

APCA DPCA APCA DPCA

\begin{tabular}{lcccccc} 
4-Item & Low & Mean & 0.083 & 0.060 & 0.156 & 0.055 \\
Auction & Synergy & (std. error) & $(0.016)$ & $(0.020)$ & $(0.026)$ & $(0.020)$ \\
& High & Mean & 0.083 & 0.060 & 0.167 & 0.058 \\
& Synergy & (std. error) & $(0.018)$ & $(0.019)$ & $(0.033)$ & $(0.021)$ \\
\hline \multirow{2}{*}{ 6-Item } & Low & Mean & 0.106 & 0.068 & 0.413 & 0.064 \\
Auction & Synergy & (std. error) & $(0.016)$ & $(0.019)$ & $(0.069)$ & $(0.018)$ \\
& High & Mean & 0.105 & 0.080 & 0.445 & 0.073 \\
& Synergy & (std. error) & $(0.015)$ & $(0.025)$ & $(0.069)$ & $(0.021)$ \\
\hline \multirow{4}{*}{ 12-Item } & Low & Mean & 2.633 & 1.888 & 88.953 & 0.759 \\
Auction & Synergy & (std. error) & $(0.224)$ & $(0.256)$ & $(16.523)$ & $(0.101)$ \\
& High & Mean & 2.648 & 2.035 & 90.384 & 0.818 \\
& Synergy & (std. error) & $(0.212)$ & $(0.382)$ & $(17.879)$ & $(0.140)$ \\
\hline
\end{tabular}

Notes: Times reported are generated using the Timing function in

Mathematica, and are the elapsed time from the first round to the end of the last round in each auction. Descending auctions use the same bid shaves as used above with no bidding error incorporated.

Table 5: Computation Times for Three-Bidder Environment

Of course, the results in Table 5 and Table 6 do not imply that every possible auction environment would require more computation time if implemented as a APCA rather than as a DPCA. Clearly, in the case where all bidders are fully revealing, the number of bids collected by the APCA includes all those submitted in the DPCA, so the APCA problem size is always larger. However for given price incrementing/decrementing rules and a particular bidding strategy, one could construct a contrary example where, in the effort to determine the auction winners, more high valued bids would be accumulated during a DPCA than low valued bids in the comparable APCA, producing a higher computation time in the DPCA. tested. 
Time take to complete auction (measured in seconds)

Myopic bidding Reveal-all bidding

APCA DPCA APCA DPCA

\begin{tabular}{lcccccc} 
4-Item & Low & Mean & 0.122 & 0.069 & 0.304 & 0.067 \\
Auction & Synergy & (std. error) & $(0.017)$ & $(0.020)$ & $(0.045)$ & $(0.020)$ \\
& High & Mean & 0.126 & 0.070 & 0.326 & 0.068 \\
& Synergy & (std. error) & $(0.022)$ & $(0.020)$ & $(0.055)$ & $(0.020)$ \\
\hline \multirow{2}{*}{ 6-Item } & Low & Mean & 0.168 & 0.086 & 1.039 & 0.076 \\
Auction & Synergy & (std. error) & $(0.021)$ & $(0.021)$ & $(0.138)$ & $(0.019)$ \\
& High & Mean & 0.189 & 0.102 & 1.236 & 0.093 \\
& Synergy & (std. error) & $(0.028)$ & $(0.028)$ & $(0.191)$ & $(0.023)$ \\
\hline \multirow{7}{*}{ 12-Item } & Low & Mean & 7.184 & 3.767 & 539.4 & 1.381 \\
Auction & Synergy & (std. error) & $(0.479)$ & $(0.612)$ & $(99.11)$ & $(0.227)$ \\
& High & Mean & 7.687 & 4.213 & 571.6 & 1.526 \\
& Synergy & (std. error) & $(0.881)$ & $(0.799)$ & $(57.11)$ & $(0.242)$ \\
\hline
\end{tabular}

Notes: Times reported are generated using the Timing function in

Mathematica, and are the elapsed time from the first round to the end of the last round in each auction. Descending auctions use the same bid shaves as used above with no bidding error incorporated.

Table 6: Computation Times for Six-Bidder Environment

But these value/strategy environments would need to be highly structured: they very rarely occur in randomly generated value sets with myopic bidding. The environment constructed by KLM is one reasonable structural representation of a combinatorial auction environment that requires competitive bidders to coalesce through aggressive bidding in order to win. We think it a fair representation of a large genre of combinatorial resource allocation problems extant in the real world. 


\subsection{Collusion: Evidence from previous literature}

Because there has been very little use of combinatorial auctions in both the laboratory and in the real world, there is limited evidence supporting collusive bidding behavior. There is, however, significant evidence of collusion in the Simultaneous Ascending Auction (SAA), a close relative of the APCA proposed by Milgrom (2000).

There exist several instances of collusive bidding behavior in real world multi-unit auctions. For a thorough summary of these cases see Klemperer (2002), and Cramton and Schwartz (2000). Most examples of real world collusive bidding involve the easily detectable signaling using particular bid digits: additional auction rules can prevent this and it is not a strategy available in any clock auction.

But Brown et al. (2009) demonstrates robust tacit collusion in a laboratory setting with the SAA institution. The tacit collusion evolved in a "collusion incubator environment under the ascending prices, but failed to form, or was broken, by a descending price version of the auction. In addition, KLM report on at least one case of collusive behavior in conducting the APCA auction where all items were sold at the auctions initial starting prices.

As highlighted by Brown et al. (2009), collusion evolves in these ascending institutions because of the ability to signal other participants by using public bids and because of the ability to punish non-cooperative bidders by using retaliatory bidding to increase prices on items in which they are interested. Brown et al. (2009) shows that making bids anonymous in an ascending auction helps prevent collusive bidding, but this may not be a desirable institutional feature in the real world. ${ }^{14}$ However, in a descending auction there is simply nothing a competitor can do once a (known) rival submits his initial (high) bid. In a DPCA a single bidder who bids on the entire package of all items stops the auction immediately. If implicitly collusive behavior allows prices to fall low enough any bidder can place a full coverage bid with no retaliation possible. A bid submitted on less than the entire package reserves those items for the bidder at those prices unless alternative overlapping packages

\footnotetext{
${ }^{14}$ In wireless spectrum auctions the FCC is required to report bidder IDs on provisionally winning bids in each round.
} 
that better fit the other bids are introduced. The strategic problem for implicit colluders becomes much more difficult to solve. The collusion breaking nature of descending prices in multi-unit auctions suggests the DPCA will perform well in this dimension.

\section{Discussion}

Iterative clock auctions provide useful price feedback for bidders in complex combinatorial environments. We outline three main concerns with the APCA institution, the relevant package problem, computational burden, and susceptibility to collusion, and conclude that the DPCA improves in all these areas. Because the sheer number of possible combinations of items in combinatorial environments the cost associated with value elicitation and the strategic incentives when submitting bids, bidders are likely to submit a small number of bids in each round of the auction and let prices guide them to their most profitable packages/items. Experiments by KLM and Scheffel et al. (2012) document this myopic bidding behavior in laboratory APCA environments. This myopic bidding behavior can cause some environments to produce low efficiency allocations. These environments occur because bidders efficiency relevant packages are not their most profitable ones at low prices and later in the auction when prices get high enough they are no longer able to submit bids on their relevant packages. The descending prices of the DPCA do not create this blocking problem, and therefore generally lead to more efficient allocations. Our simulations confirm this result: using myopic bidding agents the DPCA produces average efficiencies that are higher (statistically significant at 1\%) than the APCA. These differences are magnified in favor of the DPCA when high synergies exist. To test the robustness of these improvements over the APCA we employ noisy bid functions for agents in the DPCA and find that while the disparity in performance is less, the DPCA still performs better than the APCA in all but one environment where the APCA performs insignificantly better at $1 \%$. In addition to the relevant package problem concerns exist on the computational burden associated with combinatorial auctions. In each round of the APCA integer optimizations are required to 
determine the optimal allocation of goods. In auctions with a large number of bidders and items this computation time can prevent auctions from completing in a reasonable amount of time. To measure this burden we record the computational time required to complete each of the thousand auctions in all institutions. The average auction time for the APCA and the DPCA are very similar when myopic bidding behavior is used by the bidding agents. However, when an all-revealing bidding strategy is used the disparity in average auction time between APCA and the DPCA grows substantially, with the DPCA requiring much less computational time. This is due to the fact that the APCA collects more bids throughout the auction rounds via low prices, resulting in a larger objective function. This disparity in computation time grows with both the number of items and number of bidders in the auction.

And finally, we lean on previous field and laboratory data to reiterate that ascending multi-unit auctions can be susceptible to collusive bidding behavior. The key components of reaching a cooperative bidding strategy are bid signaling, and retaliatory bidding. Descending price institutions greatly limit or destroy these components. Brown et al. (2009) show that robust tacit collusion can evolve in ascending price multi-unit auction environments, but does not evolve, or is destroyed by descending price auctions. Because of the collusion resistance of multi-unit descending environments we expect the DPCA to show much less susceptibility to collusion than the APCA.

It is our hope that the improved performance of the DPCA suggested by our simulations can help combinatorial auctions reach a higher level of prominence in real world use. Issues stemming from computational and bidding uncertainty have caused many to shy away from the use of combinatorial auction mechanisms and toward more traditional auctions. We find little rationale for the continued use of non-combinatorial auctions in environments with item complementarities (synergies) exist. The increased simplicity of bidding in traditional auctions is nothing the APCA or DPCA cannot provide; bidders are free to submit bids for individual items if they wish. In addition, salient mechanisms allowing bidders to submit logistic constraints can eliminate the risk of undesirable over-allocations. The use of pack- 
age bidding allows bidders to freely express interest in aggregations of items without the financial risk associated with non-package auctions that leave bidders to riskily attempt to assemble a package of individual items typically subject to a potpourri of institutional rules. Additionally, the rationale that non-package auctions function well in environments with low synergies or in environments that we can be embellished with a few pre-selected packages, leaves the auctioneer responsible to pre-determine decentralized information. While combinatorial auctions carry with them some concerns for practitioners and auctioneers it is our hope that the DPCA implemented and tested in this paper, with its innovative descending price guidance system, helps to alleviate the concerns with package auctions and allows them a wider audience of use. 


\section{References}

Ausubel, L. M., Cramton, P., Milgrom, P., 2004. Combinatorial Auctions. MIT Press, Cambridge, Ch. The Clock-Proxy Auction: A Practical Combinatorial Auction Design.

Banks, J. S., Ledyard, J. O., Porter, D. P., 1989. Allocating uncertain and unresponsive resources: an experimental approach. RAND Journal of Economics 20 (1), 1-25.

Brown, A. L., Plott, C. R., Sullivan, H. J., 07 2009. Collusion facilitating and collusion breaking power of simultaneous ascending and descending price auctions. Economic Inquiry $47(3), 395-424$.

Brunner, C., Goeree, J. K., Holt, C. A., Ledyard, J. O., February 2010. An experimental test of flexible combinatorial spectrum auction formats. American Economic Journal: Microeconomics 2 (1), 39-57.

Cramton, P., 09 1997. The fcc spectrum auctions: An early assessment. Journal of Economics \& Management Strategy 6 (3), 431-495.

Cramton, P., Schwartz, J. A., May 2000. Collusive bidding: Lessons from the fcc spectrum auctions. Journal of Regulatory Economics 17 (3), 229-52.

Fujishima, Y., Leyton-Brown, K., Shoham, Y., 1999. Taming the computational complexity of combinatorial auctions: Optimal and approximate approaches. Proceedings of IJCAI99, Stockholm.

Goeree, J. K., Holt, C. A., September 2010. Hierarchical package bidding: A paper \& pencil combinatorial auction. Games and Economic Behavior 70 (1), 146-169.

Harrison, G. W., September 1989. Theory and misbehavior of first-price auctions. American Economic Review 79 (4), 749-62.

Kagel, J. H., Lien, Y., Milgrom, P., 2010. Ascending prices and package bidding: A theoretical and experimental analysis. American Economic Journal: Microeconomics 2, 160-85. 
Klemperer, P., Winter 2002. What really matters in auction design. Journal of Economic Perspectives 16 (1), 169-189.

Kwasnica, A. M., Ledyard, J. O., Porter, D., DeMartini, C., March 2005. A new and improved design for multiobject iterative auctions. Management Science 51 (3), 419-434.

McAfee, R. P., McMillan, J., Winter 1996. Analyzing the airwaves auction. Journal of Economic Perspectives 10 (1), 159-175.

Milgrom, P., April 2000. Putting auction theory to work: The simultaneous ascending auction. Journal of Political Economy 108 (2), 245-272.

Porter, D., Rassenti, S. J., Roopnarine, A., Smith, V., 2003. Combinatorial auction design. Proceedings of the National Academy of Sciences 100 (19), 11153-57.

Rassenti, S., Smith, V., Bulfin, R., Autumn 1982. A combinatorial auction mechanism for airport time slot allocation. Bell Journal of Economics 13 (2), 402-417.

Rothkopf, M. H., Pekec, A., Harstad, R. M., August 1998. Computationally manageable combinatorial auctions. Management Science 44 (8), 1131-1147.

Scheffel, T., Ziegler, G., Bichler, M., December 2012. On the impact of package selection in combinatorial auctions: An experimental study in the context of spectrum design. Experimental Economics 15 (4), 667-692.

Vickrey, W., 03 1961. Counterspeculation, auctions, and competitive sealed tenders. Journal of Finance 16 (1), 8-37. 